\title{
Tomography inversion using velocity models represented by radial basis functions
}

Daina Popic Bendoraitis $\left(^{*}\right)^{1}$, Luiz Alberto Santos ${ }^{1,2}$, Webe João Mansur ${ }^{3}$ - Petrobras ${ }^{1}$, Universidade Federal Fluminense $(\mathrm{UFF})^{2}$, Universidade Federal do Rio de Janeiro (UFRJ-COPPE) ${ }^{3}$.

Copyright 2019, SBGf - Sociedade Brasileira de Geofísica

This paper was prepared for presentation during the $16^{\text {th }}$ International Congress of the Brazilian Geophysical Society held in Rio de Janeiro, Brazil, 19-22 August 2019

Contents of this paper were reviewed by the Technical Committee of the $16^{\text {th }}$ International Congress of the Brazilian Geophysical Society and do not necessarily represent any position of the SBGf, its officers or members. Electronic reproduction or storage of any part of this paper for commercial purposes without the written consent of the Brazilian Geophysical Society is prohibited.

\section{Abstract}

Tomography is today the standard tool in velocity model building. It is a paramount part of the seismic method, whose purpose is to obtain accurate images of the interior of the planet. In this work, a traveltime tomography based on the discretization of the velocity model by radial basis functions is proposed. The forward modelling is done with an algorithm based on the resolution of the eikonal equation by finite differences. The inverse problem estimates the coefficients of the radial basis functions. Radial basis functions can represent velocity models with a reduced number of parameters and nodes can be positioned without prior information. The results of the experiments show that the developed inversion tool is able to retrieve the parameters of the models represented by these functions.

\section{Introduction}

Since 1970 decade, seismic tomography has been developed (Nolet et al., 2008). Bois et al. (1972) introduced inter-well transit time inversion. Bishop et al. (1985) presented a tomographic method to estimate reflectors depth and seismic velocity from reflection data.

The objective of tomographic inversion is to estimate subsurface velocity distribution, based in measures of transit time of transmission, refraction or reflection (Jones, 2010).

Two main steps compose a tomographic problem. The first one is the forward problem that aims to calculate the transit time and rays trajectory through a velocity model. In this work, forward modeling was carried out with an Eikonal based algorithm. The second step is the inverse problem that estimates the velocity model parameters. In this work the Gauss-Newton method was used together with Conjugate Gradient (CG) as the solver.

We tested the use of a radial basis function (RBF) to represent the velocity field. The use of RBF allows a reduced number of parameters to describe the model that adds the important benefit of computational reduction cost. Circular anomalies are very well represented and RBF can be used to represent geologic features.

\section{Theory}

According to Aster et al. (2013), the relationship between the data collected in the field and the model is generically represented by:

$$
G(m)=d,
$$

if the data is discrete, $d=\left[d_{1}, d_{2}, \ldots, d_{m}\right]^{T}$ is the data parameter vector and, in the present work, corresponds to the transit times. $m=\left[m_{1}, m_{2}, \ldots, m_{n}\right]^{T}$ is the velocity model parameters vector. The matriz $G_{m \times n}$ is the operator that relates time and velocity. It represents the physical phenomenon of the seismic wave propagation. The forward problem consists of finding $d$ given $m$ and $G$. The obtained data is called calculated or synthetic data $\left(d^{\text {calc }}\right)$. The inverse problem aims to estimate the parameter model $m$ for a known $d$.

Inverse problems in geophysics are usually ill-posed because the data is not sufficient to estimate in a unique and stable way the distribution of the physical properties in subsurface (Silva et al., 2001). The tomographic problem is also non-linear, since the operator $G$ is a function of the parameters of the model $m$.

Tomography aims to obtain a velocity model that minimizes a functional given by the difference between the transit times calculated in the forward modeling $\left(d^{\text {calc }}=G(m)\right)$ and observed data $\left(d^{o b s}\right)$, called residuals:

$$
r=d^{o b s}-G(m) \text {. }
$$

We can formulate minimization problem mathematically as:

$$
\min _{m} \gamma(m)
$$

where $m$ is the model parameters vector. The function $\gamma(m)$ is called objective function and corresponds to a measure of misfit of the residual vector. In this work, norm2 is used:

$$
\gamma(m)=\frac{1}{2}\|r\|_{2}^{2}
$$

RBFs give rise to a class of interpolation functions within the so-called meshfree methods. They use a set of nodes scattered in both the domain and the boundaries of the problem, and do not require a priori information to establish a relationship between the nodes (Liu and $\mathrm{Gu}, 2005$ ).

A RBF is a finite linear combination of base functions with radial symmetry relative to its center (Buhmann, 2003). The symmetry takes the form $\phi\left(\|.\|_{2}\right)$ where $\|.\|_{2}$ denotes the Euclidean norm:

$$
\Phi(x)=\phi\left(\|.\|_{2}\right)=\phi(r) .
$$


The independent variable is the Euclidean distance between the center of a base function and the points scattered on the problem domain. The form of $\Phi(x)$ is:

$$
\Phi(x)=\sum_{k=1}^{n} \lambda_{k} \phi\left(\left\|x-c_{k}\right\|_{2}\right),
$$

where $\lambda_{i}$ are the coefficients of the base function and $c_{k}$ are the centers, where the $n$ nodes scattered by the domain are located. Considering $\Phi\left(x_{i}\right)=f_{i}$, in matrix form we get:

$$
H \lambda=f,
$$

where the coefficients $\lambda_{k}$ are the unknowns obtained with the resolution of the linear system and

$$
H=\left[\begin{array}{cccc}
\phi\left(\left\|x_{1}-c_{1}\right\|_{2}\right) & \phi\left(\left\|x_{1}-c_{2}\right\|_{2}\right) & \cdots & \phi\left(\left\|x_{1}-c_{n}\right\|_{2}\right) \\
\phi\left(\left\|x_{2}-c_{1}\right\|_{2}\right) & \phi\left(\left\|x_{2}-c_{2}\right\|_{2}\right) & \cdots & \phi\left(\left\|x_{2}-c_{n}\right\|_{2}\right) \\
\vdots & & \ddots & \vdots \\
\phi\left(\left\|x_{m}-c_{1}\right\|_{2}\right) & \phi\left(\left\|x_{m}-c_{2}\right\|_{2}\right) & \ldots & \phi\left(\left\|x_{m}-c_{n}\right\|_{2}\right)
\end{array}\right] .
$$

The index $m$ corresponds to the number of points of the original function to be interpolated.

The study of the RBFs began in Hardy (1971) with the purpose of representing a topographic surface by means of the interpolation of scattered points, using a multiquadric function. Recently, in the area of data inversion in geophysics, Peters et al. (2017) present a methodology for adaptive parametrization for FWI, using RBFs. Dahlke et al. (2017) use a sparse representation based on RBFs to represent the implicit surface of salt bodies and developed a new formulation of the Full Waveform Inversion (FWI) objective function. Kadu et al. (2017) used a level-set function represented by an RBF to modify the geometry of a salt body during a FWI inversion process.

We chose the two-dimensional Gaussian RBF to discretize the velocity model for this work:

$$
v(x, z)=\sum_{i=1}^{n} A_{i} e^{-\frac{1}{2}\left[\frac{\left(x-x_{i}\right)^{2}}{\sigma_{x_{i}}^{2}}+\frac{\left(z-z_{i}\right)^{2}}{\sigma_{z_{i}}^{2}}\right]},
$$

where $A_{i}$ are parameters related to the height of the curve's peak of the Gaussian, $\sigma_{x_{i}}$ and $\sigma_{z_{i}}$ are parameters related to the shape of the function in the $x$ and $z$ directions, similar to the standard deviation used in statistic, and $x_{i}$ and $z_{i}$ are the coordinates of the Gaussian center.

\section{Method}

The experiment considered a synthetic velocity model with dimensions of $2000 \mathrm{~m} \times 2000 \mathrm{~m}$, in the coordinates $x$ (horizontal) and $z$ (vertical). Two hypothetical vertical wells are positioned, with the sources located at coordinate $x=0.0$ and the receivers at coordinate $x=2000.0$. The observed data, derived from the synthetic model, is generated with the same algorithm used to generate the calculated data.

A background model with vertical velocity gradient was generated with 9 Gaussian radial basis functions (Fig. 1). Table 1 displays the value of the $A$ parameter for each node and the $\sigma$ parameter is the same for all points: $\sigma_{x}=8000$ and $\sigma_{z}=1000$. Anomalies were added to this model, also represented by Gaussian FBR. The objective of the inversion is to determine the parameter $A$ of each anomaly.

The tomographic problem was solved by the GaussNewton optimization method, where a search direction and a step size are found. It starts from an initial model $m_{0}$ and the problem is replaced by a sequence of linear least squares problems, solved in successive iterations and whose final solution converges to the solution of the original nonlinear problem (Heath, 2002; Menke, 2012).

The update expression of the model is given by:

$$
J^{T}(m)^{i} J^{T}(m)^{i} \Delta m^{i}=-J^{T}(m)^{i} \Delta d^{i},
$$

where $J^{T}(m)^{i}$ is the Jacobian, or sensitivity matrix, of the $i$ th iteration and $J^{T}$ denotes its transpose. A small perturbation in the data $\Delta d^{i}$ is related to a small perturbation in the model $\Delta m^{i}$. The model perturbation $\Delta m_{i}$ is used to update the model parameters vector $m_{i+1}$. Each entry of the $J^{T}$ matrix is given by:

$$
J^{T}=\frac{\partial G\left(m_{q}\right)}{\partial m_{j}} .
$$

For each source, one generates a travel time matrix, from which the trajectory and transit time of each ray is calculated.

In each iteration of the program a new Jacobian matrix is calculated and the result of the inversion corresponds to an updating direction of the model $\Delta m$. This direction is then normalized and multiplied by a step size. Step sizes decrease during iterations.

According to ray theory, the travel time of a wave generated at a source and arriving at a receiver can be obtained by means of the eikonal equation:

$$
|\nabla T(\boldsymbol{x})|^{2}=\frac{1}{v(\boldsymbol{x})^{2}}
$$

where $\boldsymbol{x}=(x, z)$ represents the position vector and $x$ and $z$ are the horizontal and vertical coordinates, respectively, $v(\boldsymbol{x})$ is the medium velocity and $T(\boldsymbol{x})$ represents the arrival time of a wavefront at position $\boldsymbol{x}$.

The forward problem was solved using the algorithm developed by Podvin and Lecomte (1991). These authors proposed a method to calculate the transit time at any position of a spatial mesh, solving the eikonal equation using finite differences. The output of the algorithm is a transit time matrix.

Ray tracing is calculated from receivers to the source, in the opposite direction to the time gradient of the transit time matrix.

A module was implemented to verify, for each ray, if any of its segments are within an influence support proportional to the $\sigma$ parameter of the anomaly under consideration. If the ray crosses that region, the sensitivity coefficient is calculated. Otherwise, the coefficient is nullified. The objective is to discard coefficients of rays that has little influence of the anomaly, as illustrated in figure 2 . 


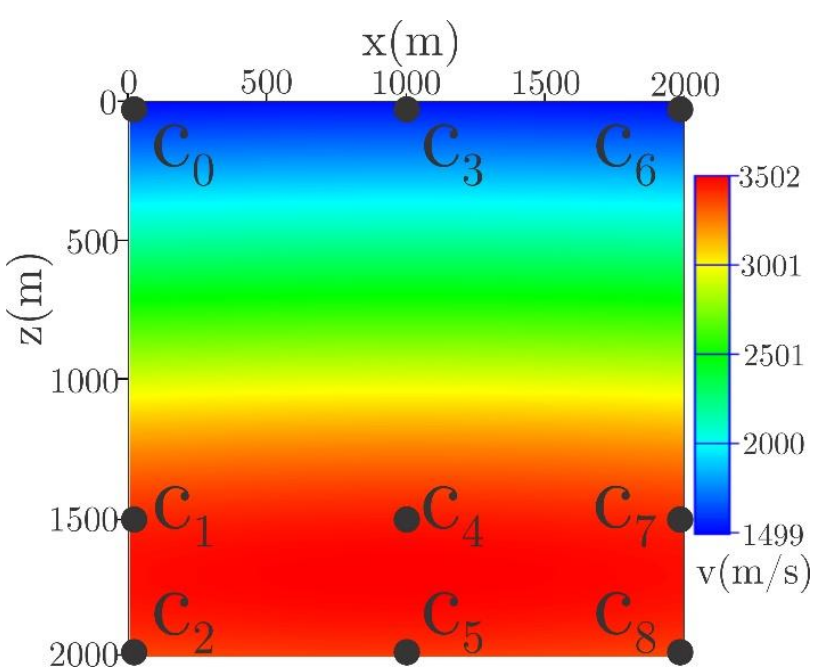

Figure 1 - Background model. Circles in black show the location of Gaussian FBR centers.

Table 1 - Values of the A parameter for the background model

\begin{tabular}{|l|l|l|l|}
\hline$A_{i}=265.0 \mathrm{~m} / \mathrm{s}$ & $C_{0}$ & $C_{3}$ & $C_{6}$ \\
\hline$A_{i}=450.0 \mathrm{~m} / \mathrm{s}$ & $C_{1}$ & $C_{4}$ & $C_{7}$ \\
\hline$A_{i}=700.0 \mathrm{~m} / \mathrm{s}$ & $C_{2}$ & $C_{5}$ & $C_{8}$ \\
\hline
\end{tabular}

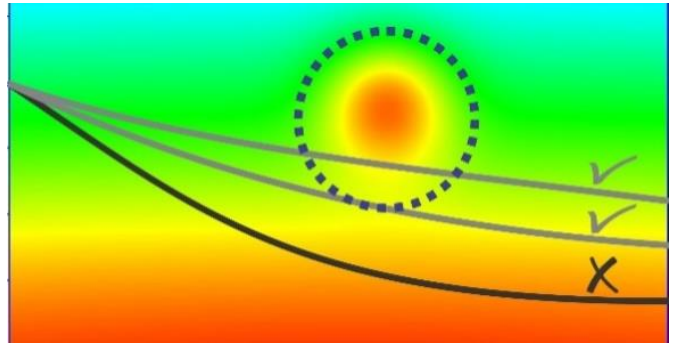

Figure 2 - The doted circumference around the anomaly represents the support region. Rays that pass within this region have their coefficient of sensitivity calculated otherwise they become null.

\section{Results}

In this section we present inversion results for velocity models containing anomalies represented by the Gaussian RBF. The objective of the experiment is to obtain the correct value of the $A$ parameter of these anomalies.

The examples highlights the flexibility of parameterization with these functions.

The first two experiments consist of two anomaly points with $\sigma_{x}=\sigma_{z}=100$ and an additional one where there is no anomalous value, i.e., only the background model. The positions and values of the $A$ parameter of these points can be checked in table 2 and table 3 . A total of 40 sources housed between $z=0.0$ and $z=1950.0$ at interval of $50 \mathrm{~m}$ and 40 receivers from the coordinate $z=0.0$ and $z=1950.0$ with interval of $50 \mathrm{~m}$ are used. Thus, 1600 rays are defined. The objective is to evaluate if the inversion is able to recover the correct value of the $A$ parameter in the positions indicated. The figure 3 shows the result for an initial model very close to the background model, while the figure 4 shows the result for an initial model closer to the target. Tables 2 and 3 show the target, initial value and the result obtained for the $A$ parameter. The graph of figure 5 shows the reduction of the objective function for each iteration, comparing the 2 experiments.
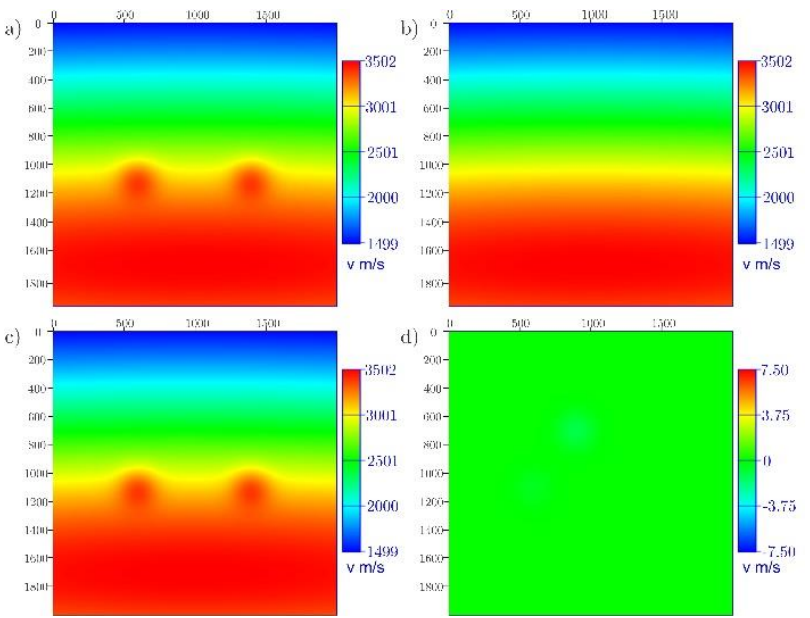

Figure 3 - Experiment 1: a) Target; b) Initial model close to the background model; c) Result; d) Error.

Table 2 - Data for experiment 1 with initial model close to the background model.

\begin{tabular}{|c|c|c|c|c|}
\hline $\begin{array}{c}\text { Location } \\
\mathrm{x}(\mathrm{m})\end{array}$ & $\begin{array}{c}\text { Location } \\
\mathrm{z}(\mathrm{m})\end{array}$ & $\begin{array}{c}\text { Target } \\
(\mathrm{m} / \mathrm{s})\end{array}$ & $\begin{array}{c}\text { Initial } \\
\text { value } \\
(\mathrm{m} / \mathrm{s})\end{array}$ & $\begin{array}{c}\text { Result } \\
(\mathrm{m} / \mathrm{s})\end{array}$ \\
\hline 600.0 & 1100.0 & 300.0 & 1.0 & 300.559 \\
\hline 1400.0 & 1100.0 & 300.0 & 1.0 & 300.002 \\
\hline 900.0 & 700.0 & 0.0 & 1.0 & 1.18312 \\
\hline
\end{tabular}
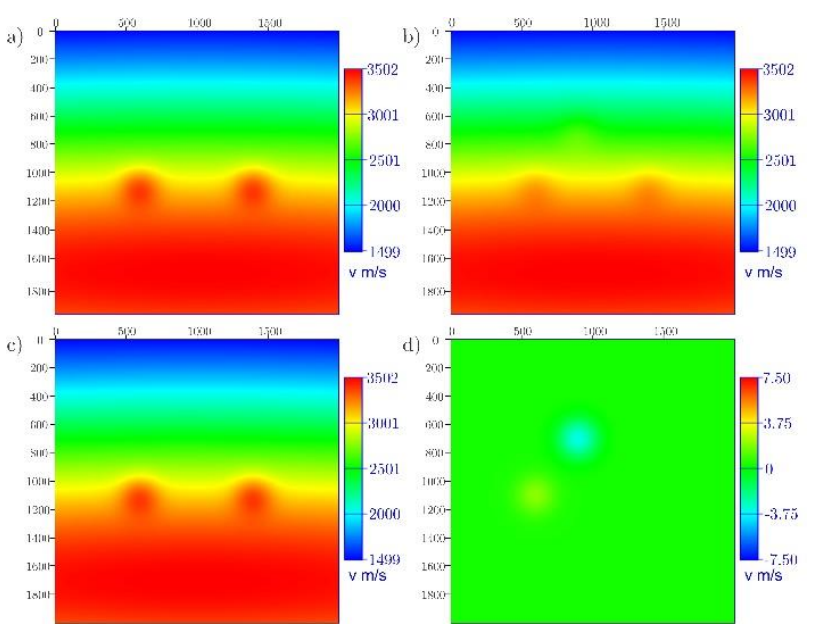

Figure 4 - Experiment 2: a) Target; b) Initial model with perturbation towards the correct model; c) Result; d) Error. 
Table 3 - Data for experiment 2 with perturbation towards the correct model.

\begin{tabular}{|c|c|c|c|c|}
\hline $\begin{array}{c}\text { Location } \\
\mathrm{x}(\mathrm{m})\end{array}$ & $\begin{array}{c}\text { Location } \\
\mathrm{z}(\mathrm{m})\end{array}$ & $\begin{array}{c}\text { Target } \\
(\mathrm{m} / \mathrm{s})\end{array}$ & $\begin{array}{c}\text { Initial } \\
\text { value } \\
(\mathrm{m} / \mathrm{s})\end{array}$ & $\begin{array}{c}\text { Result } \\
(\mathrm{m} / \mathrm{s})\end{array}$ \\
\hline 600.0 & 1100.0 & 300.0 & 120.0 & 298.303 \\
\hline 1400.0 & 1100.0 & 300.0 & 120.0 & 299.998 \\
\hline 900.0 & 700.0 & 0.0 & 120.0 & 3.60441 \\
\hline
\end{tabular}

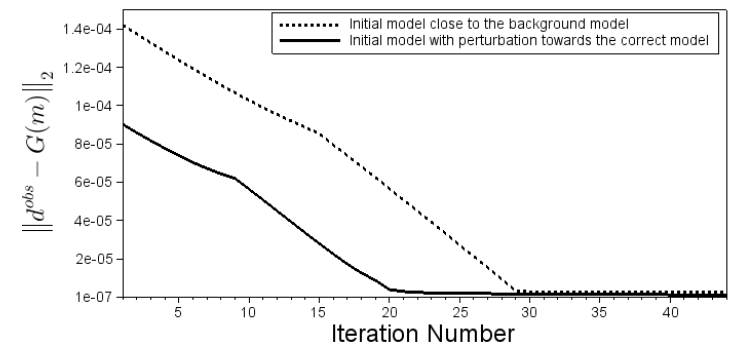

Figure 5-Objective function value for each iteration comparing experiments 1 and 2.

The next two experiments are composed of a horizontal feature of lower velocity than the background. The goal is to simulate a lens or a slower velocity layer of rocks. It consists of 3 points with $\sigma_{x}=200$ and $\sigma_{z}=100$. The support region for the inversion is $200 \mathrm{~m}$. The position of the points is shown in the tables 4 and 5 . The acquisition device is composed by 10 sources from the coordinate $z=0.0$ with an interval of $200 \mathrm{~m}$ and 10 receivers from the coordinate $z=0.0$ equally spaced at each $200 \mathrm{~m}$. A hundred rays is needed with this device. First an initial model close to the background model is used (Fig. 6) and, in the sequence, an initial model with perturbation towards the target (Fig. 7). The tables 4 and 5 present the data. The graph of figure 8 compares the objective function for each iteration, for both cases.

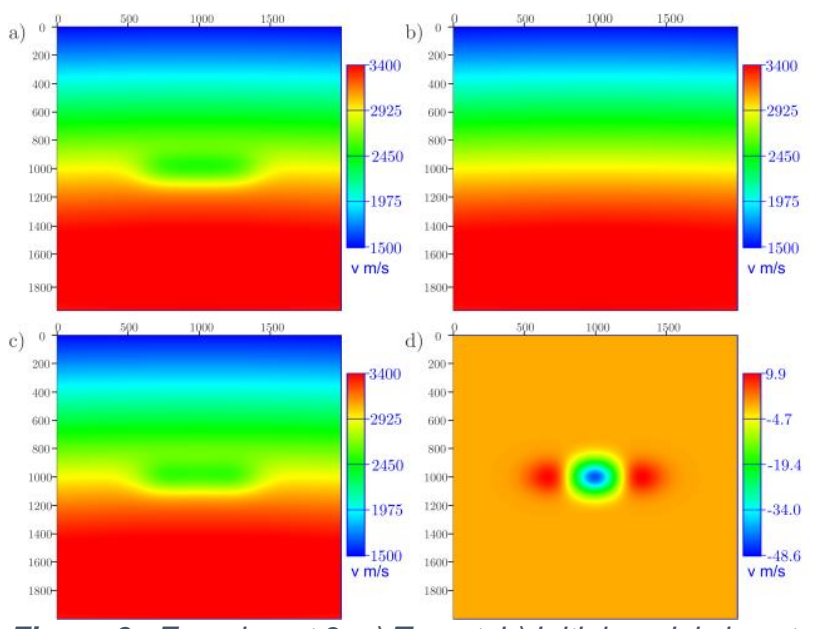

Figure 6 - Experiment 3: a) Target; b) Initial model close to the background model; c) Result; d) Error.
Table 4 - Data for experiment 3 with initial model close to the background model.

\begin{tabular}{|c|c|c|c|c|}
\hline $\begin{array}{c}\text { Location } \\
\mathrm{x}(\mathrm{m})\end{array}$ & $\begin{array}{c}\text { Location } \\
\mathrm{z}(\mathrm{m})\end{array}$ & $\begin{array}{c}\text { Target } \\
(\mathrm{m} / \mathrm{s})\end{array}$ & $\begin{array}{c}\text { Initial } \\
\text { value } \\
(\mathrm{m} / \mathrm{s})\end{array}$ & $\begin{array}{c}\text { Result } \\
(\mathrm{m} / \mathrm{s})\end{array}$ \\
\hline 750.0 & 1000.0 & -300.0 & -1.0 & -315.205 \\
\hline 1000.0 & 1000.0 & -300.0 & -1.0 & -249.122 \\
\hline 1250.0 & 1000.0 & -300.0 & -1.0 & -315.550 \\
\hline
\end{tabular}

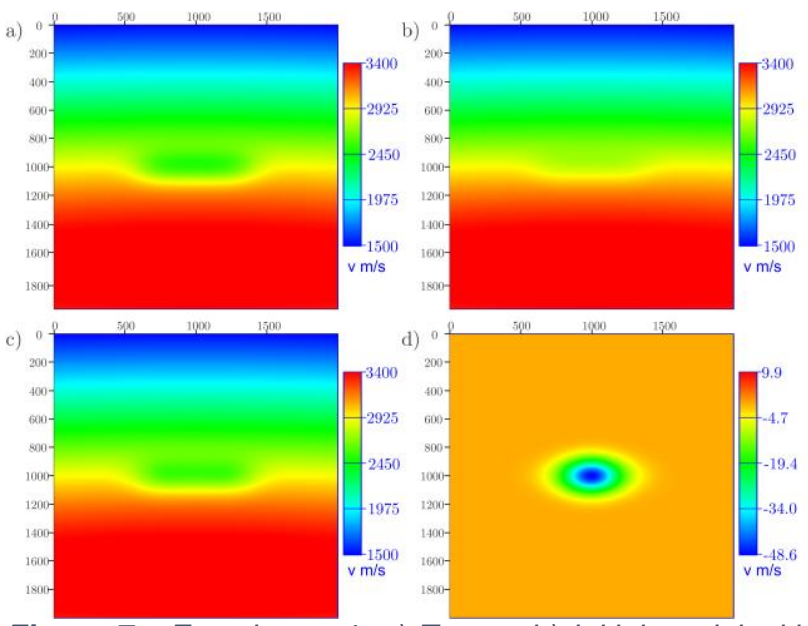

Figure 7 - Experiment 4: a) Target; b) Initial model with perturbation towards the correct model; c) Result; d) Error.

Table 5 - Data for experiment 4 with perturbation towards the correct model.

\begin{tabular}{|c|c|c|c|c|}
\hline $\begin{array}{c}\text { Location } \\
\mathrm{x}(\mathrm{m})\end{array}$ & $\begin{array}{c}\text { Location } \\
\mathrm{z}(\mathrm{m})\end{array}$ & $\begin{array}{c}\text { Target } \\
(\mathrm{m} / \mathrm{s})\end{array}$ & $\begin{array}{c}\text { Initial } \\
\text { value } \\
(\mathrm{m} / \mathrm{s})\end{array}$ & $\begin{array}{c}\text { Result } \\
(\mathrm{m} / \mathrm{s})\end{array}$ \\
\hline 750.0 & 1000.0 & -300.0 & -100.0 & -297.468 \\
\hline 1000.0 & 1000.0 & -300.0 & -100.0 & -252.647 \\
\hline 1250.0 & 1000.0 & -300.0 & -100.0 & -296.715 \\
\hline
\end{tabular}

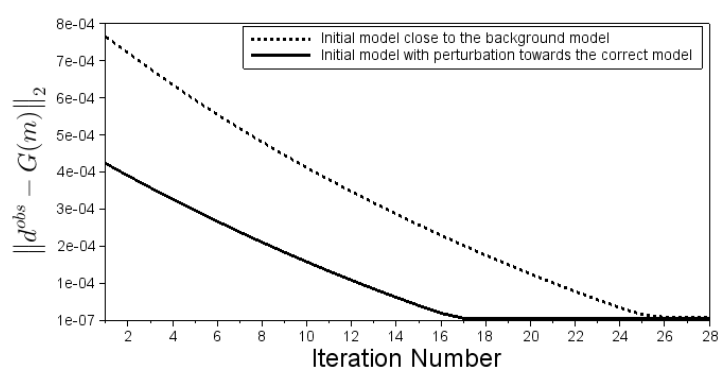

Figure 8 - Objective function value for each iteration comparing experiments 3 and 4.

According to the results of experiments 1 and 2, we observe that the inversion approach described here is able to approximate the correct values of the parameter $A$. From the graph of the figure 5 we find that both examples have a similar value for the objective function after some iterations, although, for the initial model close to the background it is necessary more iterations to find this result. 
For experiments 3 and 4, it is shown that is possible to recover the desired feature, although the amplitudes do not exactly match the target. The center point has larger amplitude than the lateral points for both cases, with a smaller error for experiment 4 . The $200 \mathrm{~m}$ support region adopted has influence in the result, since we can see that one point compensates the amplitudes of the others in the process of inversion.

\section{Conclusions}

Gaussian FBR proved to be a useful tool for representing velocity model. For smooth models, few parameters can be used with storage and computational resources savings. The technique is suitable for generating initial models. In addition, there is flexibility in parameterization, since there is no need for a priori information to position the function centers. One possibility is to concentrate points in regions of higher velocity contrast and to space them in the more monotonous portions, which would lead to a more optimized inversion process.

Circular features can be complex to discretize using a regular grid. In this case, it would be necessary very small cells. Radial based functions represent this kind of feature very well. Some examples are volcanic rocks, bodies of salt, lenses and diapers.

Tomographic inversion based on the Gaussian FBR may be a useful tool to find velocity anomalies. It presents great flexibility in parameterization and identifies anomalies where they are present, without changing the model when they are absent. These results suggest its utility as a mechanism of quality control or search for anomalous regions in velocity models.

\section{Acknowledgments}

This study was financed in part by the Coordenação de Aperfeiçoamento de Pessoal de Nível Superior - Brasil (CAPES) - Finance Code 001 and CNPq, Conselho Nacional de Desenvolvimento Científico e Tecnológico Brasil, which the authors thank and also thank to PETROBRAS.

\section{References}

Aster, R. C., B. Borchers, and C. H. Thurber, 2013, Parameter estimation and inverse problems, 2 ed.: Academic Press.

Bishop, T., K. Bube, R. Cutler, R. Langan, P. Love, J. Resnick, R. Shuey, D. Spindler, and H. Wyld, 1985, Tomographic determination of velocity and depth in laterally varying media: Geophysics, 50, 903-92.

Bois, P., M. La Porte, M. Lavergne, and G. Thomas, 1972, Well-to-well seismic measurements: Geophysics, 37, 471480 .

Buhmann, M. D., 2003, Radial basis functions: theory and implementations: Cambridge University Press.
Dahlke, T., B. Biondi, and R. Clapp, 2017, Using the hessian of a radial basis formulation for level set inversion: SEP-Report, 170.

Hardy, R. L., 1971, Multiquadric equations of topography and other irregular surfaces: Journal of geophysical research, 76, 1905-1915.

Heath, M. T., 2002, Scientific computing: McGraw-Hill.

Jones, I. F., 2010, Tutorial: Velocity estimation via raybased tomography: first break, 28, 45-52.

Nolet, G., et al., 2008, A breviary of seismic tomography: Imaging the Interior.

Kadu, A., T. van Leeuwen, andW. A. Mulder, 2017, Salt reconstruction in full-waveform inversion with a parametric level-set method: IEEE Transactions on Computational Imaging, 3, 305-315.

Liu, G.-R., and Y.-T. Gu, 2005, An introduction to meshfree methods and their programming: Springer Science \& Business Media.

Menke, W., 2012, Geophysical data analysis: Discrete inverse theory, 3 ed.: Academic press.

Peters, F. C., Junior, E. F. F., Mansur, W. J., et al., 2017, "An adaptive meshless parameterization for full waveform inversion", Engineering Analysis with Boundary Elements, v. 83 , pp. 113-122.

Podvin, P., and I. Lecomte, 1991, Finite difference computation of traveltimes in very contrasted velocity models: a massively parallel approach and its associated tools: Geophysical Journal International, 105, 271-284.

Silva, J. B., W. E. Medeiros, and V. C. Barbosa, 2001, Pitfalls in nonlinear inversion: Pure and Applied Geophysics, 158, 945-964. 